\title{
A novel emboli protection cannula during cardiac surgery: In vitro results
}

\author{
Liran Shani, MD, Oved Cohen, MD, Ziv Beckerman, MD, Idit Avrahami, PhD, Rony-Reuven Nir, PhD, and \\ Gil Bolotin, MD, PhD
}

Objective: Intraoperative cerebral events are mainly caused by emboli generated by operative manipulation of the aorta. This study aimed to delineate the distribution profiles of emboli with 2 widely used cannulae and a third novel research cannula that simultaneously produces forward flow and backward suction to extract emboli from the distal aorta during cardiac surgery.

\begin{abstract}
Methods: The current in vitro study used a silicone model of the aortic arch and branches. The main outcome measure was the distribution profile of embolic particles of different sizes to the aortic branches; 2 commercial cannulae and a third novel cannula with and without suction were used. The research cannula was examined at different suction levels and the amount of particles retrieved was measured.
\end{abstract}

Results: For the research curved-tip cannula, most of the small emboli were released into the brachiocephalic trunk in the model $(P<.05)$. For the straight-tip cannula, most of the small emboli were released into the descending aorta $(P<.05)$. Regarding the commercial curved-tipped cannula, most of the small emboli were released into the brachiocephalic trunk $(47.14 \% \pm 4.78 \% ; P<.05)$ and the medium and large emboli were predominantly released into the descending aorta. Using suction, the research cannula retrieved most of the emboli released into the aorta for all particle sizes $(50 \%-83 \% ; P<.05)$.

Conclusions: A straight-tip cannula may be safer in terms of cerebral embolic consequences during cardiac surgery. Furthermore, the use of the research aortic cannula may be beneficial in the cardiac surgery setting by reducing the postoperative risk for stroke. (J Thorac Cardiovasc Surg 2014;148:668-75)

The risk of neurologic injury is significant for patients undergoing cardiac surgery and is associated with increased mortality, morbidity, hospital costs, and impaired quality of life. ${ }^{1}$ Cardiac surgery involves a wide spectrum of neurologic injuries, including ischemic stroke, occurring in $1.5 \%$ to $5.2 \%$ of patients; encephalopathy, affecting $8.4 \%$ to $32 \%$ of patients; and neurocognitive dysfunction, manifested in $20 \%$ to $30 \%$ of patients 1 month after surgery. ${ }^{2,3}$ Embolism is considered the main mechanism of neurologic injury. ${ }^{4}$

Emboli can be divided into macro and micro categories according to size. Macroemboli occlude flow in arteries 200 $\mu \mathrm{m}$ or greater in diameter, whereas microemboli occlude flow in smaller arteries, arterioles, and capillaries. ${ }^{5}$ Thirty to fifty percent of perioperative strokes detected with brain imaging are caused by a cerebral macroembolism likely arising from the ascending aorta. ${ }^{6-8}$ Encephalopathy and

From the Department of Cardiac Surgery, Rambam Health Care Campus, Haifa, Israel.

Disclosures: Dr Bolotin served as a consultant for Cardiogard, and possesses stock options in Cardioguard. All other authors have nothing to disclose with regard to commercial support.

Received for publication July 2, 2013; revisions received Jan 6, 2014; accepted for publication Jan 7, 2014; available ahead of print Feb 9, 2014.

Address for reprints: Gil Bolotin, MD, PhD, Department of Cardiac Surgery, Rambam Health Care Campus, Haifa, POB 9602, 31096, Israel (E-mail: g_bolotin@rambam.health.gov.il).

$0022-5223 / \$ 36.00$

Copyright (c) 2014 by The American Association for Thoracic Surgery

http://dx.doi.org/10.1016/j.jtcvs.2014.01.001 neurocognitive dysfunction are believed to result primarily from cerebral microembolisms, ${ }^{5,9}$ which are either gaseous or particulate in composition. Gaseous emboli can arise from an open left-sided cardiac chamber or from air entrained into the cardiopulmonary bypass (CPB) circuit. $^{2} \mathrm{An}$ increased rate of embolic material released into the cerebral circulation has been detected during various aortic manipulations performed in cardiac surgery (eg, aortic crossclamp placement and removal, aortic cannulation, and side-biting clamping for proximal coronary artery anastomosis). ${ }^{10-12}$

The aim of the current study was to assess 2 commercial cannulae and a third new aortic cannula with and without suction. The novel cardiac cannula simultaneously induces forward flow and backward suction to remove solid and gaseous emboli from the distal aorta on their release during cardiac surgery.

\section{MATERIALS AND METHODS \\ Description of the Commercial and Research Aortic Cannulae}

The current study compared the properties of 3 different aortic cannulae; 2 commercial cannulae and the research cannula. The 2 commercial cannulae included (1) a straight-tip 24-Fr aortic cannula (DLP straight tip arterial cannula; Medtronic, Inc, Minneapolis, Minn); and (2) a curvedtip $\left(45^{\circ}\right)$ 24-Fr aortic cannula (ARS024CS; Edwards Lifesciences, Irvine, Calif). The research cannula was a curved-tip $\left(60^{\circ}\right) 24-\mathrm{Fr}$ aortic cannula (CardioGard; BEL Bioengineering Laboratories, Cantù, Italy).

The novel research cannula comprises 2 hollow cylinders (Figure 1); the first cylinder is a standard main forward-flow tube administering 


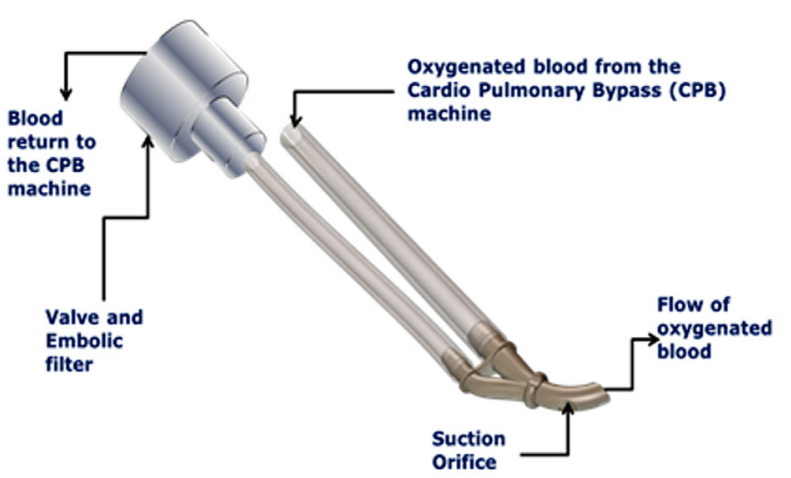

\begin{tabular}{||l|l|}
\hline Characteristics & $\begin{array}{l}\text { Specificati } \\
\text { on } / \text { Value }\end{array}$ \\
\hline Tip Size & $24 \mathrm{Fr}$ \\
\hline $\begin{array}{l}\text { Tip } \\
\text { Length }\end{array}$ & $20 \mathrm{~mm}$ \\
\hline Tip Form & Curved \\
\hline
\end{tabular}
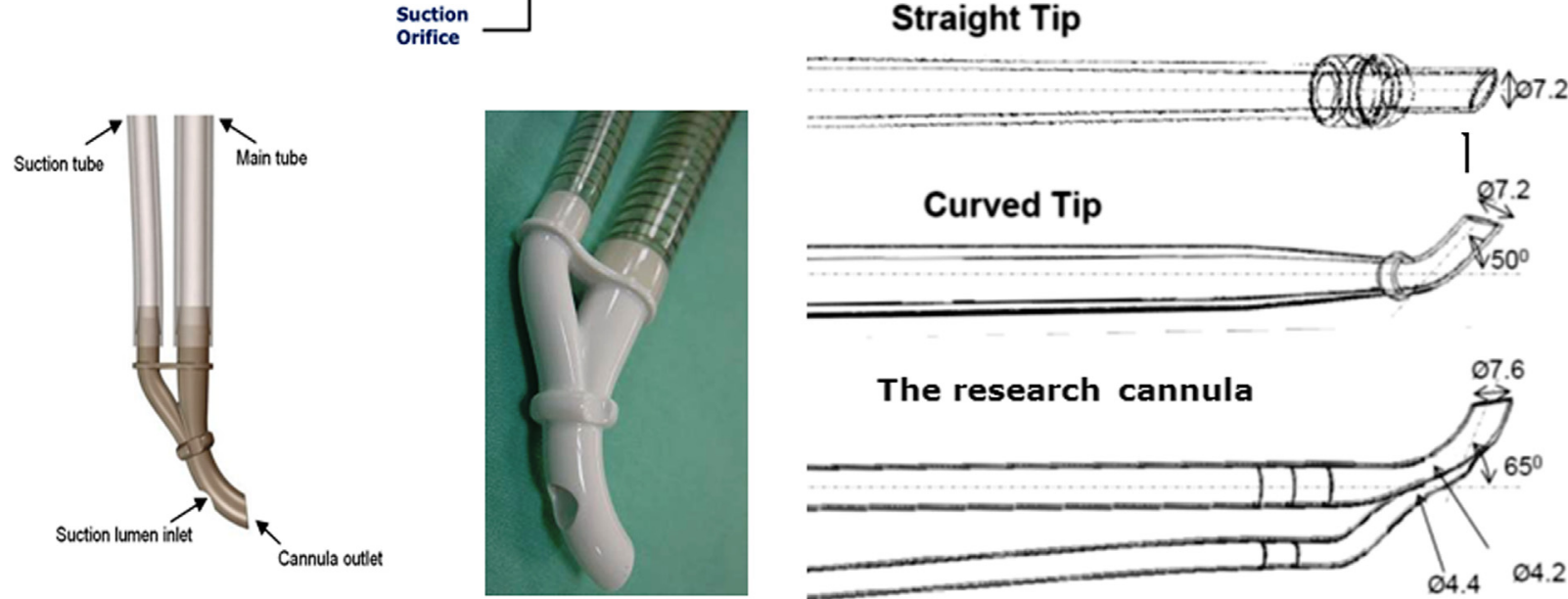

FIGURE 1. The cannulae used in the study.

arterial blood into the aorta from the bypass machine. The second cylinder, which is attached to an existing bypass vent port, is a novel element located posterior to the main tube. Its function is to facilitate blood and particle suction by directing the blood back to the reservoir of the bypass machine, while the evacuated emboli material is eliminated through a filter.

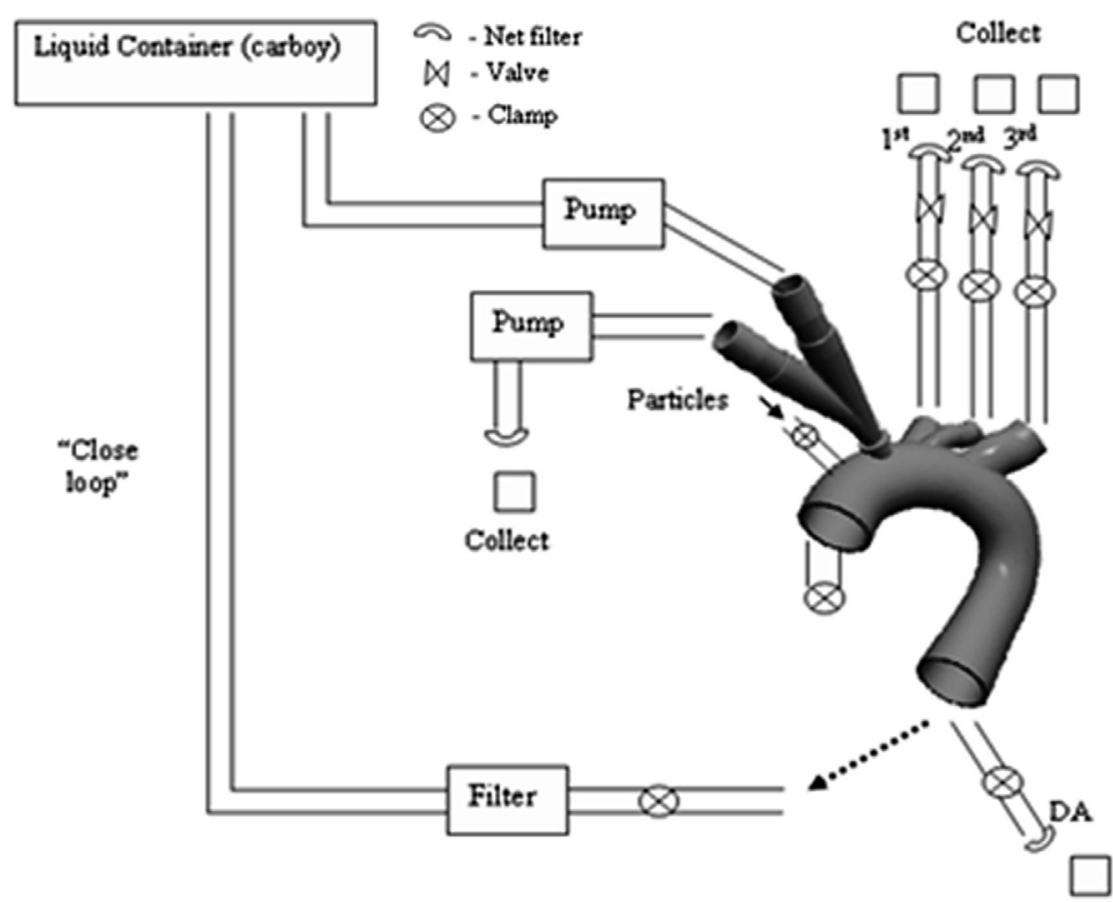

FIGURE 2. The silicone in vitro model of the aorta. The upper 3 exits simulate (1) the brachiocephalic trunk, (2) the left common carotid artery, and (3) the left subclavian artery. The lower exit simulates the descending aorta (DA). 

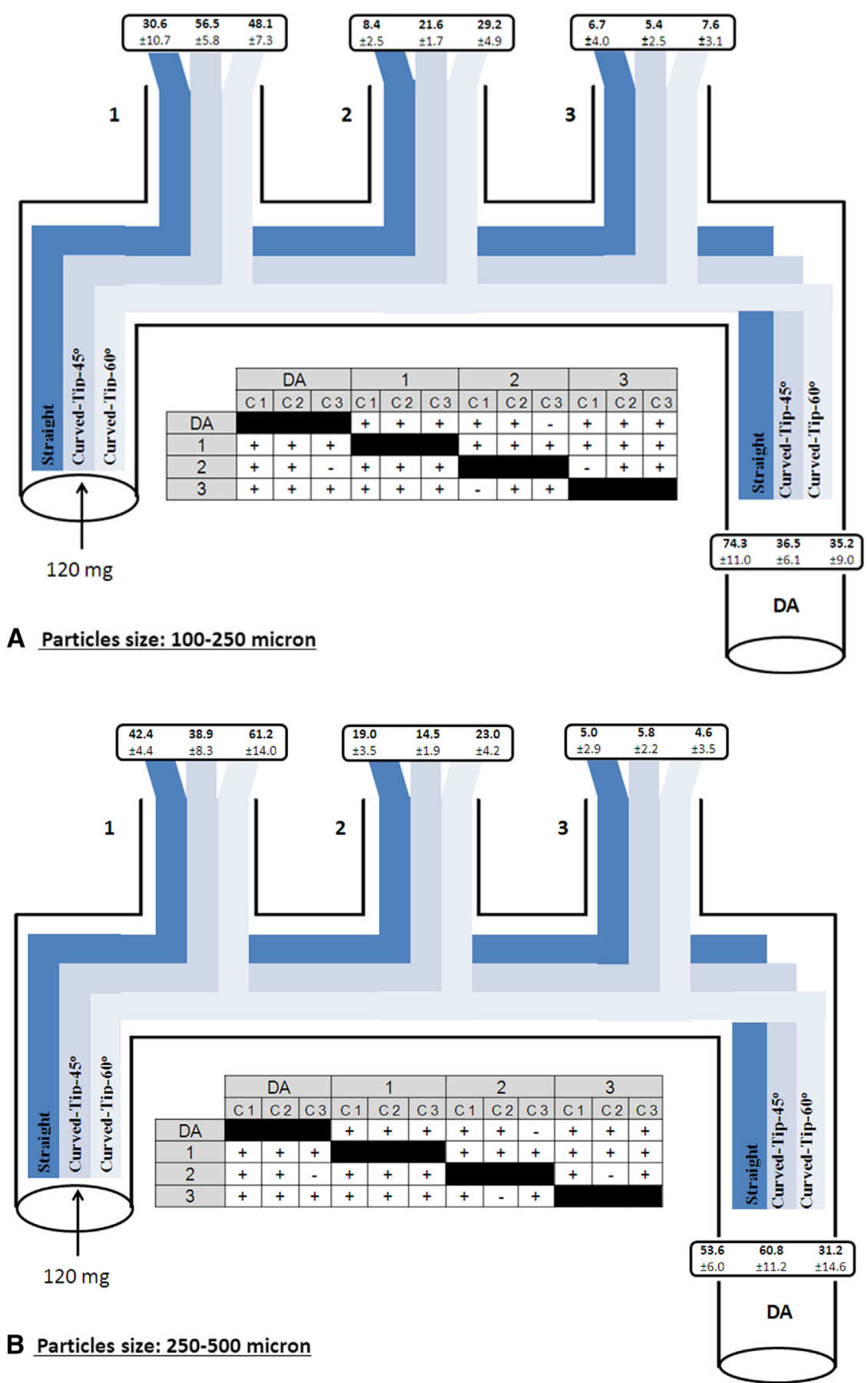

FIGURE 3. A-C, Description and comparison of the amount (in milligrams) of emboli released in the various branches of the experimental model without suction, according to the size of the particles and the type of cannula (namely, [C1] straight-tip cannula, [C2] curved-tip $45^{\circ}$ cannula, and [C3] curved-tip $60^{\circ}$ cannula, CardioGard). The upper 3 exits simulate (1) the brachiocephalic trunk, (2) the left common carotid artery, and (3) the left subclavian artery. The lower exit simulates the descending aorta $(D A)$. The tables represent significant differences $(P<.05)$, denoted by + , between the weight of emboli filtered by the various cannulae at the various exits.

\section{Aortic In Vitro Model}

A silicone model of the aorta with its 3 arch branches was constructed (Figure 2). A fourth exit simulated the descending aorta. A 70- $\mu$ m diameter filter was placed at each exit to capture solid and gaseous embolic material. A mixed fluid composed of $80 \%$ water and $20 \%$ glycerin was used to simulate blood.

\section{Injection Procedure}

Bovine bone was ground into particles of 100 to $1000 \mu \mathrm{m}$. Solid emboli material $(120 \mathrm{mg})$ of 3 different sizes were injected: (1) 100 to $250 \mu \mathrm{m}$ in diameter in the first session; (2) 250 to $500 \mu \mathrm{m}$ in the second session; and (3) 500-1000 $\mu \mathrm{m}$ in the third session. Embolic material was placed between 2 clamps in the proximal silicone aorta. Both clamps 


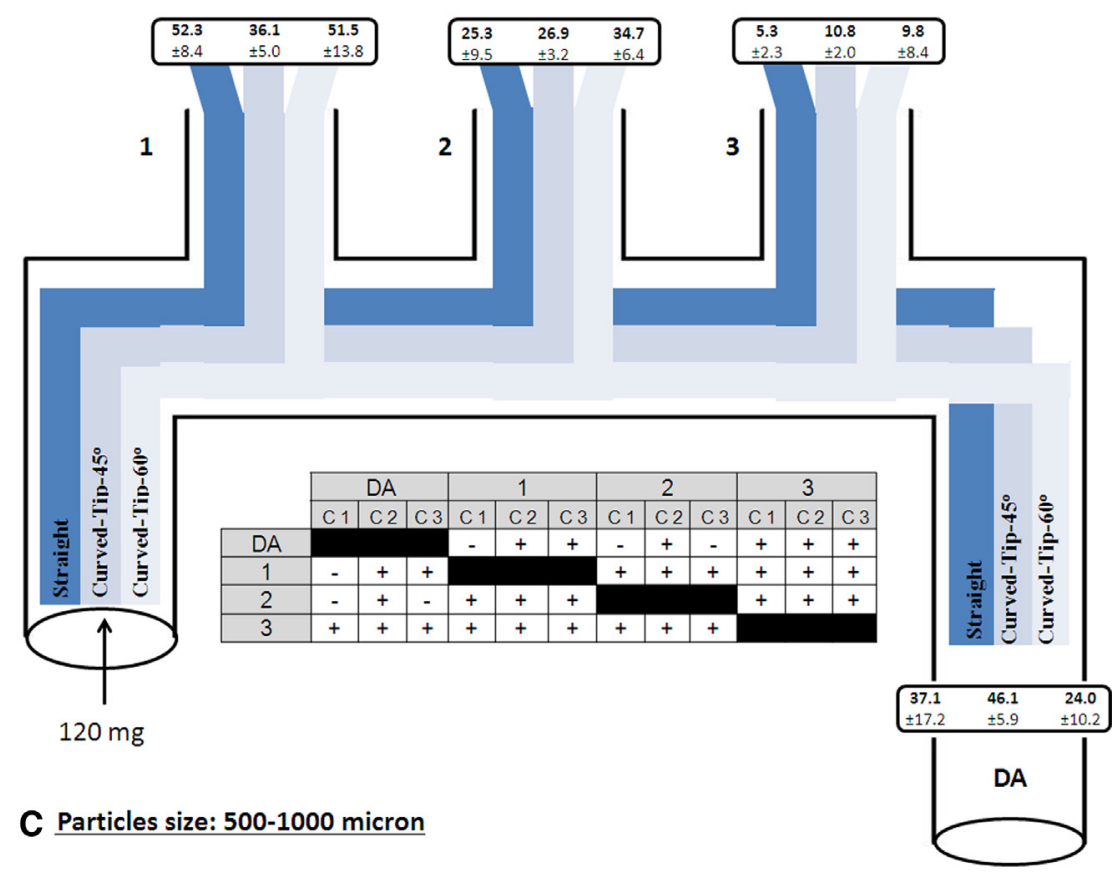

FIGURE 3. (continued).

were released simultaneously. Flow was set to $4 \mathrm{~L} / \mathrm{min}$ for nonsuction conditions and $4.5 \mathrm{~L} / \mathrm{min}$ for the suction experiments. Every injection procedure was repeated 8 times. At the end of every cycle, the filters were washed and the embolic material at every exit was weighed. The research cannula was examined under different levels of suction flow $(0,0.5,1$, and $1.5 \mathrm{~L} / \mathrm{min})$.

\section{Statistical Analysis}

All analyses were conducted using the Statistical Package for the Social Sciences, Version 19 (SPSS Inc., Chicago, Ill). Data are presented as means \pm standard deviation (SD). Analyses of variance and post hoc Tukey tests were used to compare the amount of emboli material retrieved at the various exits across the different particle sizes using the various cannulae.

\section{RESULTS}

\section{Description of the Distribution Profiles of the Emboli} for the Various Cannulae

For the straight-tip cannula, most of the emboli were released into the descending aorta for the small and medium particles $(74.3 \pm 11.0 \mathrm{mg}, 53.6 \pm 6.0 \mathrm{mg}$, respectively; $P<.05$ ); for the large particles, the emboli were predominantly released into the brachiocephalic trunk $(52.3 \pm 8.4$ $\mathrm{mg} ; P<.05)$. Figure 3 describes and compares the weight of the emboli released in the various branches of the experimental model without suction, according to the size of particles and type of cannula.

For the curved-tipped cannula (no. 2), most of the small particles were released into the brachiocephalic trunk $(56.5 \pm 5.8 \mathrm{mg} ; P<.05)$; the medium and large particles were predominantly released into the descending aorta $(60.8 \pm 11.2 \mathrm{mg}, 46.1 \pm 5.9 \mathrm{mg}$, respectively; $P<.05)$.
The distribution profile of the research cannula was similar for all particle sizes; most emboli were released into the brachiocephalic trunk $(48.1 \pm 7.3 \mathrm{mg}$, $61.2 \pm 14.0 \mathrm{mg}, 51.5 \pm 13.8 \mathrm{mg}$, respectively; $P<.05$ ).

\section{The Research Cannula: Comparison of the Various Suction Levels}

The use of the suction function of the research cannula, regardless of its intensity and for all particle sizes, enabled the retrieval of a considerable amount of emboli from the aorta. Figure 4 describes the weight of emboli released in the various branches of the experimental model with suction, according to the size of the released particles. The amount of filtered emboli significantly increased with higher suction levels, for all particle sizes: $60.1 \pm 5.4 \mathrm{mg}$ to $100.1 \pm 6.6 \mathrm{mg}$ for the small particles; $62.3 \pm 4.6 \mathrm{mg}$ to $95.0 \pm 4.2 \mathrm{mg}$ for the medium particles; and $60.0 \pm 3.6 \mathrm{mg}$ to $95.3 \pm 5.9 \mathrm{mg}$ for the large particles $(P<.05)$. For each suction level, the amount of particles retrieved was comparable for the different particle sizes $(P>.05)$.

\section{Comparison of the Straight, Curved Commercial and Research (With Suction) Cannulae}

Using the research cannula, the weight of emboli caught in the brachiocephalic trunk and left common carotid artery was significantly reduced under all suction conditions $(P<.05)$. Figure 5 depicts the weight of emboli filtered by the brachiocephalic trunk and left common carotid artery using the various cannulae by particle 

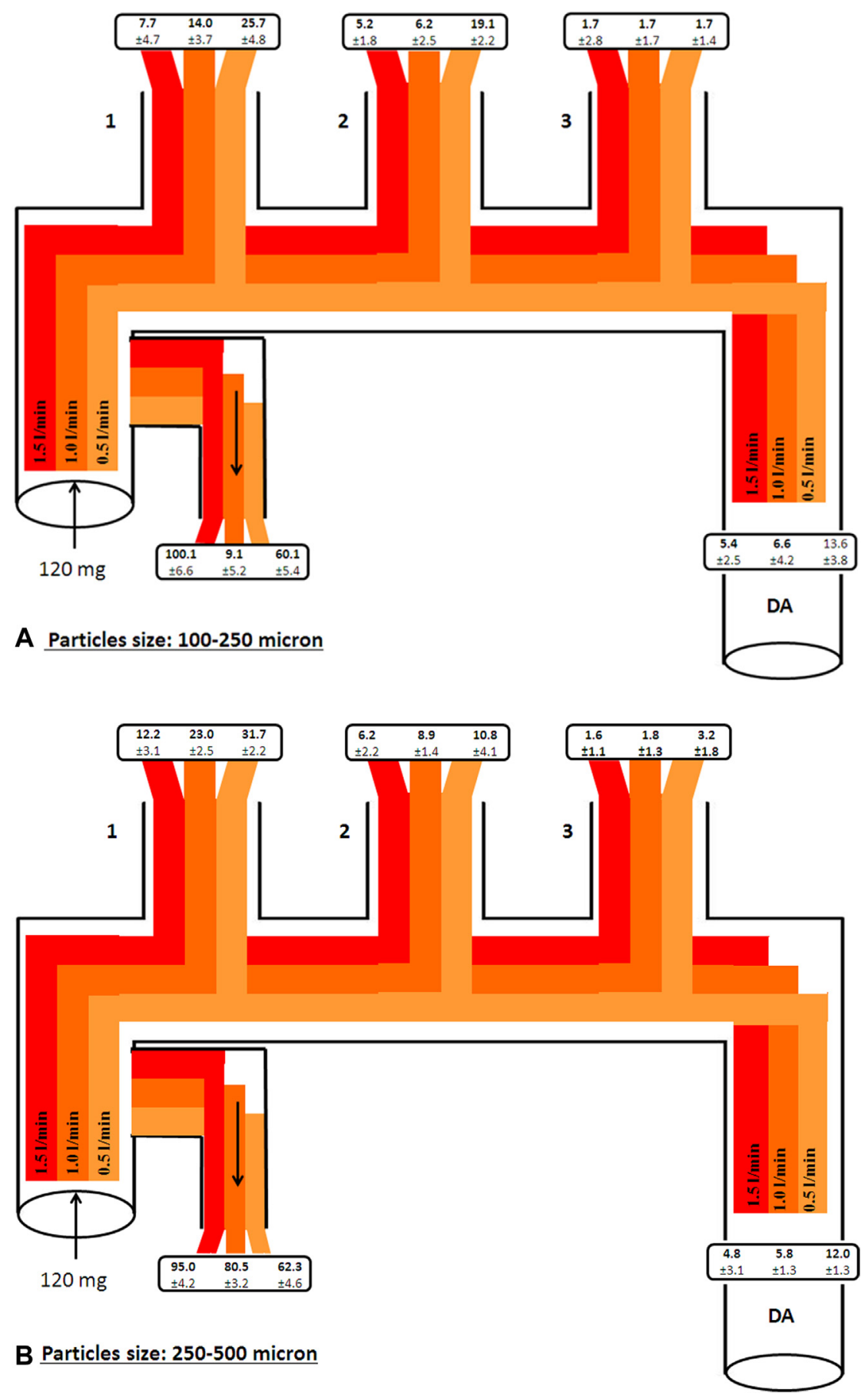

FIGURE 4. A and B, The amount of emboli released (in milligrams) the various branches of the experimental model (curved-tip $60^{\circ}$ cannula, CardioGard) with suction (1.5 L/min, $1.0 \mathrm{~L} / \mathrm{min}, 0.5 \mathrm{~L} / \mathrm{min})$, according to size of particles in the novel research cannula. The amount of filtered emboli significantly increased with higher suction levels for all particle sizes $(P<.0001)$. The upper 3 exits simulate $(1)$ the brachiocephalic trunk, (2) the left common carotid artery, and (3) the left subclavian artery. The lower right exit simulates the descending aorta $(D A)$ and the lower left exit simulates the suction function.

size: (1) for the small particles (100-250 $\mu \mathrm{m})$ there was a significant decrease in the amount of embolic material that was caught in these exits (straight tip, highest amount; curved tip $60^{\circ}$, smallest amount); (2) for the medium $(250-500 \mu \mathrm{m})$ and large $(500-1000 \mu \mathrm{m})$ particles, a comparable amount of embolic material was caught using the straight-tip and curved-tip $45^{\circ}$ cannulae, and compared with each of these cannula, the curved-tip $60^{\circ}$ cannula captured a significantly smaller amount of emboli. 


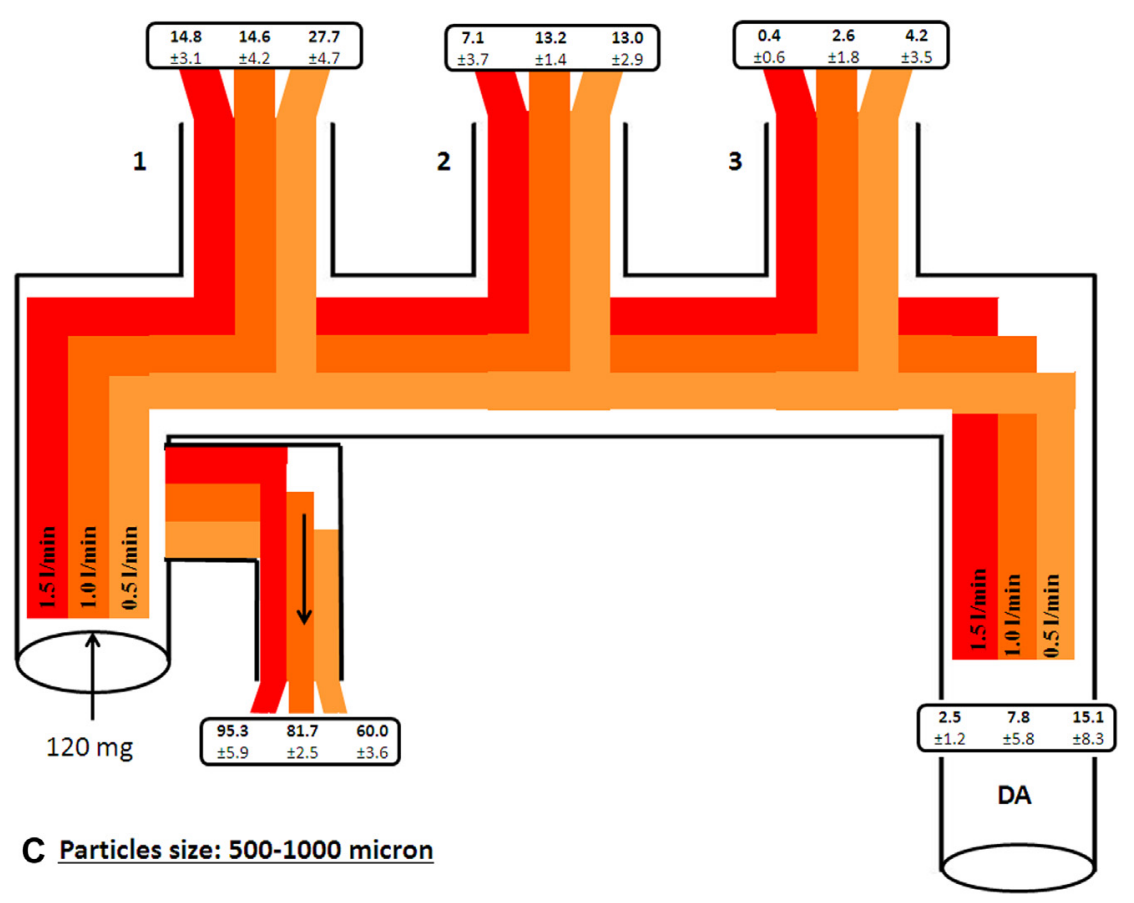

FIGURE 4. (continued).

\section{DISCUSSION}

The main finding of the current study is that the novel research aortic cannula enabled the retrieval of most of the embolic material released into the model aorta, suggesting its potential for dramatically reducing the postoperative risk for stroke.

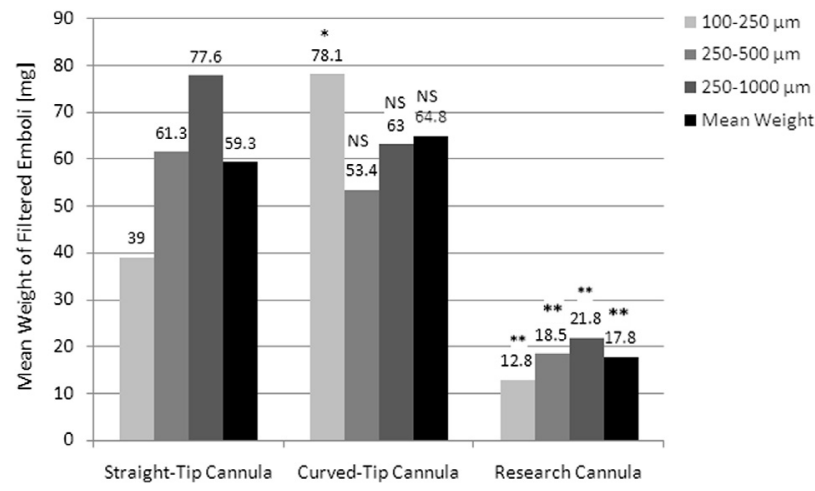

FIGURE 5. The amount of emboli (in milligrams) filtered by the brachiocephalic trunk and left common carotid artery using the various cannulae (namely, [1] straight-tip cannula, [2] curved-tip $45^{\circ}$ cannula, and [3] curved-tip $60^{\circ}$ cannula, CardioGard, $1.5 \mathrm{~L} / \mathrm{min}$ ), according to size $(100-250 \mu \mathrm{m}, 250-500 \mu \mathrm{m}$, and 500-1000 $\mu \mathrm{m})$. Comparisons were conducted according to particle size ( 3 analyses of variance; $P<.0001$ ). Tukey post hoc comparisons. *Significant difference between the curvedtip cannula and the straight-tip cannula; NS denotes a nonsignificant difference between the curved-tip cannula and the straight-tip cannula. **Significant decrease in the weight of emboli captured using the research cannula compared with the straight-tip and the curved-tip cannulae.

\section{Comparison of Emboli Distribution Profiles Between the Different Cannulae Without Suction}

The distribution profiles of the straight, curved and the novel research cannulae may be attributed to the physical properties of the cannulae; namely, the larger the angle of the cannula tip, the greater the chances of the emboli being released into the upper part of the aorta, and specifically, the first branch. The clinical significance of this observation is that the straight-tip cannula may be associated with a reduced risk of cerebral emboli. These findings are in agreement Avrahami and colleagues ${ }^{13}$ who investigated numerical models of different cannula orientations, aortic anatomies, and cannula designs. Albert and colleagues ${ }^{14}$ studied the impact of the shape of aortic end-hole cannulae on stroke occurrence and concluded that straight-tip cannulae cause significantly more frequent and more severe bilateral and posterior strokes than curved-tip cannulae. Similar conclusions were presented by White and colleagues ${ }^{15}$ who compared curved-tip cannulae with standard straight-tip cannulae.

\section{Emboli Distribution of the Novel Aortic Cannula With Suction}

The novel aortic cannula enabled the retrieval of most of the embolic material released into the model aorta, in the range of 60 to $99.6 \mathrm{mg}(50 \%-83 \%)$, for all particle sizes. This finding proved the theoretic efficacy of the cannula to reduce the risk of cerebral events during cardiac surgery. Avrahami and colleagues ${ }^{16}$ conducted 

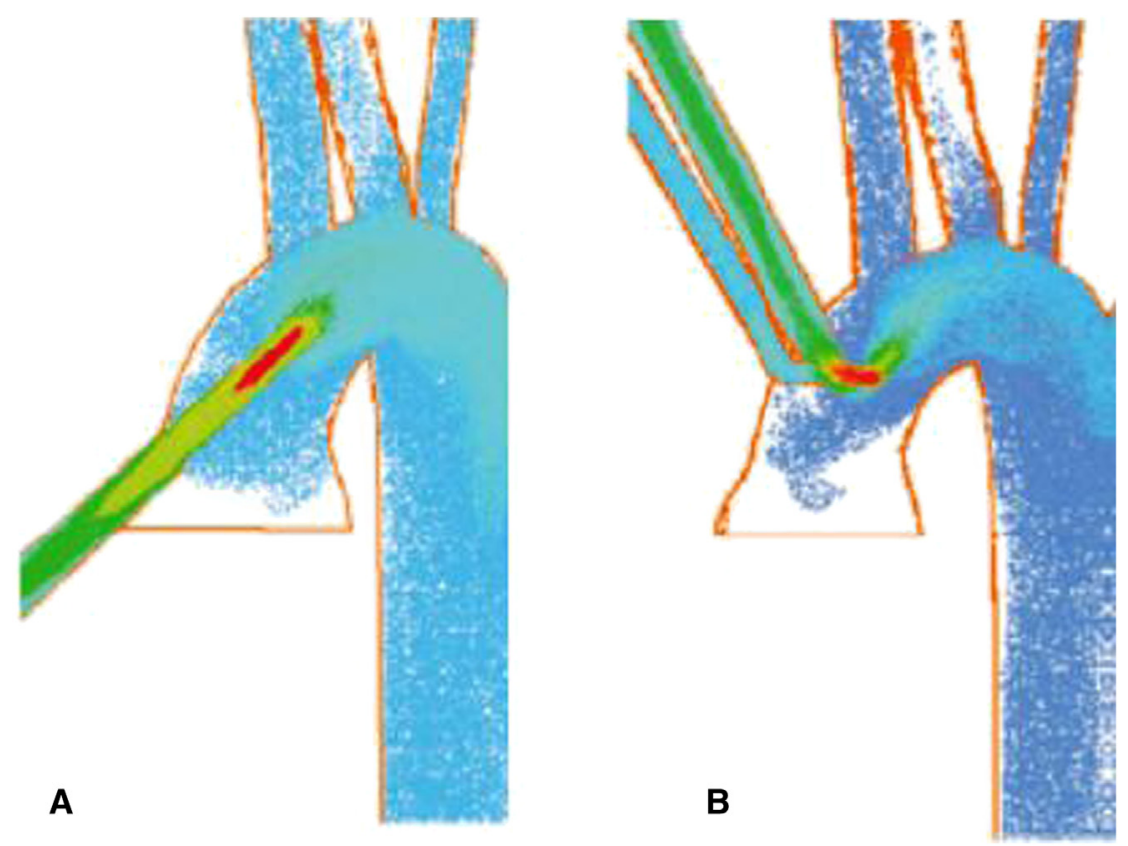

FIGURE 6. A, Blood flow stagnation between the cannula and the clamp may lead to the generation of embolic material through thrombus formation. $\mathrm{B}$, The novel cannula enables the elimination of blood flow stagnation and the removal of thrombotic material. Adapted with permission from Avrahami and colleagues. ${ }^{13}$

computerized simulations and demonstrated that compared with straight-tip and curved-tip commercial cannulae (the ones used in the current study), the novel aortic cannula was associated with a significant improvement in terms of potential risk of cerebral embolism, and similar risks for other hemodynamic complications, such as hemolysis or atheroembolism. Shani and colleagues ${ }^{17}$ investigated the novel aortic cannula in an in vivo model and reported that in terms of safety and hemocompatibility, the novel aortic cannula was comparable with the standard, widely used aortic cannula tested in the current study, and demonstrated a reduced risk of cerebral emboli.

The novel aortic cannula presented in the current study was developed for the purpose of reducing cerebral events after cardiac surgery, mainly by allowing the surgeon to perform the surgical procedure within safer limits of aortic manipulation, including crossclamp placement and removal as well as proximal anastomosis.

The capacity of the research cannula to retrieve emboli is enabled in several ways: (1) collection of embolic material coming behind the suction orifice and generated by manipulations of the aorta during the surgical procedure, as well as air and solid particles released from the heart after declamping of the aorta; (2) the cannula is eliminating embolic material generated in the space between the cannulation site and the aortic clamp as a result of stagnation of blood flow, as demonstrated by Avrahami and colleagues ${ }^{13}$ (Figure 6). This type of embolic material is usually released into the systemic circulation after declamping of the aorta.

Several types of devices used after cannulation minimize the quantity of emboli released during cardiac surgery. First, an ultrasound-based device that is placed on the aorta during surgery is used to divert the released particles from the cerebral circulation toward the descending aorta. ${ }^{18}$ Second, a percutaneously placed device inserted into the right forelimb and deployed in the aorta ${ }^{19}$ performs a similar action of diverting particles from the cerebral circulation. Third, an intra-aortic filtration device in the aortic cannula incorporates a filter that captures particulate emboli during cardiopulmonary bypass. ${ }^{20}$

In comparison with existing devices for intraoperative emboli reduction, the cannula under study is less invasive and is based on complete emboli removal rather than their deflection to the lower limbs, preventing ischemic events within the systemic circulation, including the kidneys, gastrointestinal system, and lower extremities.

Several limitations within the current study need to be addressed. First, the study did not include cycles of injected embolic material of mixed size, which may have portrayed the systemic circulation more reliably. Second, because current findings are based on an in vitro model, the ability to generalize to the clinical setting is limited. Third, the study was designed such that a fixed inflow was set but the suction level ranged from 0.5 to $1.5 \mathrm{~L} / \mathrm{min}$, leading to a corresponding variation in the net flow values. This technical matter may have had an effect on the distribution 
profile of the embolic material in the silicone model branches, such that more embolic material was captured in the suction filter as the suction level increased; however, maintaining a constant net flow would have probably given rise to lower amounts of captured emboli. Third, the current results were achieved under clamp-release conditions, relevant to a small fraction of the surgical procedure, which is significant in terms of emboli release.

In conclusion, the current findings support the use of the novel aortic cannula in cardiac surgery for the potential reduction in the rate of cerebral events. A human, multicenter, randomized, controlled clinical trial is currently under way.

\section{References}

1. Roach GW, Kanchuger M, Mangano CM, Newman M, Nussmeier N, Wolman R, et al. Adverse cerebral outcomes after coronary bypass surgery. $N$ Engl J Med. 1996;335:1857-63.

2. Hogue CW Jr, Palin CA, Arrowsmith JE. Cardiopulmonary bypass management and neurologic outcomes: an evidence-based appraisal of current practices. Anesth Analg. 2006;103:21-37.

3. Alexander KP, Anstrom KJ, Muhlbaier LH, Grosswald RD, Smith PK, Jones RH, et al. Outcomes of cardiac surgery in patients $>$ or $=80$ years: results from the National Cardiovascular Network. J Am Coll Cardiol. 2000;35:731-8.

4. Hogue CW, Gottesman RF, Stearns J. Mechanisms of cerebral injury from cardiac surgery. Crit Care Clin. 2008;24:83-98, viii-ix.

5. Blauth CI. Macroemboli and microemboli during cardiopulmonary bypass. Ann Thorac Surg. 1995;59:1300-3.

6. Blauth CI, Cosgrove DM, Webb BW, Ratliff NB, Boylan M, Piedmonte MR, et al. Atheroembolism from the ascending aorta: an emerging problem in cardiac surgery. J Thorac Cardiovasc Surg. 1992;103:1104-11.

7. Djaiani G, Fedorko L, Borger M, Mikulis D, Carroll J, Cheng D, et al. Mild to moderate atheromatous disease of the thoracic aorta and new ischemic brain lesions after conventional coronary artery bypass graft surgery. Stroke. 2004;35:e356-8.

8. Lilosky DS, Marrin CA, Caplan LR, Baribeau YR, Morton JR, Weintraub RM, et al. Determination of etiologic mechanism of strokes secondary to coronary artery bypass graft surgery. Stroke. 2003;34:2830-4.
9. Arnold JV, Blauth CI, Smith PL, Jagoe JR, Wootton R, Taylor KM Demonstration of cerebral microemboli occurring during coronary artery bypass graft surgery using fluorescein angiography. J Audiovisual Commun Med. 1990; $13: 87-90$.

10. Barbut D, Hinton RB, Szatrowski TP, Hartman GS, Bruefach M, Williams Russo P, et al. Cerebral emboli detected during bypass surgery are associated with clamp removal. Stroke. 1994;25:2398-402.

11. Rao V, Cohen G, Fedorko L, Feindel CM, Borger MA, Taylor RL, et al Decreased cerebral emboli during distal aortic arch cannulation: a randomized clinical trial. J Thorac Cardiovasc Surg. 1999;118:740-5.

12. Stump DA, Rogers AT, Hammon JW, Newman SP. Cerebral emboli and cognitive outcome after cardiac surgery. J Cardiothorac Vasc Anesth. 1996;10: 113-8.

13. Avrahami I, Dilmoney B, Hirshorn O, Brand M, Cohen O, Shani L, et al Numerical investigation of a novel aortic cannula aimed at reducing cerebral embolism during cardiovascular bypass surgery. $J$ Biomech 2013;46:354-61.

14. Albert AA, Beller CJ, Arnrich B, Walter JA, Rosendahl UP, Hetzel A, et al. Is there any impact of the shape of aortic end-hole cannula on stroke occurrence? Clinical evaluation of straight and bent-tip aortic cannulae. Perfusion. 2002;17: 451-6.

15. White JK, Jagannath A, Titus J, Yoneyama R, Madsen J, Agnihotri AK Funnel-tipped aortic cannula for reduction of atheroemboli. Ann Thorac Surg. 2009;88:551-7.

16. Avrahami I, Dilmoney B, Azuri A, Brand M, Cohen O, Shani L, et al Investigation of risks for cerebral embolism associated with the hemodynamics of cardiopulmonary bypass cannula: a numerical model. Artif Organs. 2013; 37:857-65.

17. Shani L, Cohen O, Beckerman Z, Nir RR, Bolotin G. Novel emboli protection cannula during cardiac surgery: first animal study. Asian Cardiovasc Thorac Ann. 2014;22:25-30.

18. Sauren LD, la Meir M, Bolotin G, van der Veen FH, Heijmans JH, Mess WH, et al. The EmBlocker: efficiency of a new ultrasonic embolic protection device adjunctive to heart valve surgery. Ann Thorac Surg. 2009;88:253-7.

19. Carpenter JP, Carpenter JT, Tellez A, Webb JG, Yi GH, Granada JF A percutaneous aortic device for cerebral embolic protection during cardiovascular intervention. J Vasc Surg. 2011;54:174-81.

20. Banbury MK, Kouchoukos NT, Allen KB, Slaughter MS, Weissman NJ Berry GJ, et al. Emboli capture using the EMBOL-X intraaortic filter in cardiac surgery: a multicentered randomized trial of 1289 patients. Ann Thorac Surg. $2003 ; 76: 508-15$ 